\title{
Adipose tissue at the crossroads in the development of the metabolic syndrome, inflammation and atherosclerosis
}

\author{
Tecido adiposo na encruzilhada no desenvolvimento da \\ síndrome metabólica, inflamação e aterosclerose
}

Bernardo Léo Wajchenberg ${ }^{1,2}$, Marcia Nery',

Maria Rosaria Cunha', Maria Elizabeth Rossi da Silva ${ }^{1}$

\begin{abstract}
The authors analyze insulin resistance, the metabolic syndrome and endothelial dysfunction as consequence of a common antecedent, a low grade inflammation, indicating that in obesity there is a chronically activated inflammatory state of the adipose tissue. Furthermore, the inflammatory signaling is discussed according to the adipose tissue depot, visceral or subcutaneous. Arq Bras Endocrinol Metab. 2009;53(2):145-150.

Keywords

Obesity; visceral and subcutaneous adipose tissue; low grade inflammation; atherosclerosis; metabolic syndrome
\end{abstract}

\section{RESUMO}

Os autores analisam a resistência à insulina, a síndrome metabólica e a disfunção endotelial como consequência de um antecedente comum, a inflamação de baixo nível, o que mostra que a obesidade é um estado inflamatório cronicamente ativado do tecido adiposo. Discute-se, aqui, a sinalização inflamatória de acordo com a localização do tecido adiposo subcutâneo ou visceral. Arq Bras Endocrinol Metab. 2009;53(2):145-150.

Descritores

Obesidade; tecido adiposo subcutâneo ou visceral; inflamação de baixo nível; aterosclerose; síndrome metabólica
Serviço de Endocrinologia, Hospital das Clínicas da Faculdade de Medicina da Universidade de São Paulo (HC-FMUSP), São Paulo, SP, Brasil; Centro de Diabetes e Coração, Instituto do Coração (InCor), HC-FMUSP, São Paulo, SP, Brasil

${ }^{2}$ Serviço de Endocrinologia, Hospital das Clínicas da Faculdade de Medicina da Universidade de São Paulo (HC-FMUSP), São Paulo, SP, Brasil

Correspondence to:

Bernardo Léo Wajchenberg Av. Dr. Arnaldo, 455 01246-903 - São Paulo, SP, Brasil bernarwaj@globo.com

Received in Feb/02/2009 Accepted in Feb/17/2009
$\mathrm{T}$ he clustering of dyslipidemia, hypertension and glucose intolerance, predominantly in overweight individuals, at risk of heart disease, has been received many names, including syndrome $\mathrm{X}$ and metabolic syndrome. In Reaven's original description of the syndrome, a central etiological role was attributed to insulin resistance and/or hyperinsulinemia, in part determined by obesity (1), and this assumption has become the dominant paradigm for the metabolic syndrome (2). However, there is a substantial contradiction in such association. In part because of measurement problems and the fact that though "insulin resistance" may be important, it provides an insecure foundation for the metabolic syndrome, to a level in which it is no longer considered a useful (or at least measurable) criterion. A number of associated clinical features congregate in individuals who are at increased risk of heart disease. They cluster together for a reason, and it is important to seek an explanation for this at a pathophysiological level (3).

Regarding the novel markers as responsible for associations to insulin resistance, there is a possibility that they may help to explain relations which seem biologically implausible. In particular, the coexistence of microalbuminuria with insulin resistance in diabetic and non-diabetic individuals as well as the association of insulin resistance with other measures of endothelial dysfunction are difficult to be conceptualize as a cause-andconsequence relationship. Although there is a possibility 
of a reverse etiology, whereby insulin resistance is a consequence of endothelial dysfunction, both insulin resistance and endothelial dysfunction are consequences of a common etiological mechanism (4). In a population of healthy adults, Yudkin and cols. (5) reported good correlations with metabolic syndrome variables, including insulin resistance and endothelial dysfunction, with a score of low grade inflammation derived from circulating concentrations of cytokines TNF- $\alpha$ and interleukin-6 (IL-6) and the acute-phase markers C-reactive protein (CRP) and fibrinogen, suggesting that $35 \%$ of the variance of the measures comprising the metabolic syndrome could be "explained by a state of low-grade inflammation". The findings brought, for the first time, the possibility that the association between insulin resistance and the components of the metabolic syndrome could be a consequence of their common outcomes of a low-grade inflammation state.

Furthermore, there were strong and consistent relationships with anthropometric measures of obesity and central fat distribution, as about $20 \%$ of the variance of the acute-phase markers could be explained, statistically, on the basis of adiposity (5). Many prospective studies have shown that excess of body fat in the upper (central or abdominal) part of the body is more often correlated to the features of the metabolic syndrome. In contrast, individuals with fat stored in gluteal-femoral or peripheral depots (lower-body obesity) or femaletype of fat distribution have a lower risk of morbidity from these metabolic disturbances (6). As reviewed by Karelis and cols. (7), approximately $20 \%$ of the general population can be categorized as obese but metabolically healthy, presenting low visceral fat with high body mass index (BMI) and high insulin sensitivity. Quite the opposite, $18 \%$ of the population were found to have a normal body weight or were slightly overweight (metabolically obese normal weight), with high visceral fat, low BMI, low insulin sensitivity and high liver fat displaying severe metabolic abnormalities. Recently, Stefan and cols. (8), found, in a large population, that while the measurement of visceral fat provides a powerful tool to discriminate between insulin-sensitive and insulin-resistant, subjects within normal weight and overweight range. In the obese spectrum, the predictive effect of visceral fat was relatively weak. Visceral fat was lower in obese-insulin sensitive group than the obese-insulin resistant group, but the difference was not statistically significant, maybe unexpected considering the data collected by Wajchenberg and cols. (6). Nevertheless, in agreement with our study, Stefan and cols. (8) agree that excess of fat, particularly visceral fat, when inflamed, affects insulin sensitivity in large scale; however, with increased total adiposity, factors beyond excess of visceral fat may become more important for regulating insulin sensitivity, such as the liver and the muscle.

As suggested by Yudkin and cols. $(2,5)$, a more likely paradigm for the metabolic syndrome seems to be that of adipose tissue-generated molecules initiating a state of low-grade inflammation, with the known actions of these pro-inflammatory cytokines resulting in the combined metabolic, hemodynamic and vascular consequences of this state. In this paradigm, insulin resistance merely becomes another consequence of this low grade inflammatory state.

Hotamisligil and cols. (9) were the first to describe the molecular connection between inflammation and obesity when TNF- $\alpha$, an inflammatory cytokine, was found to be expressed in adipose tissue in obese animal models, contributing to inhibition of insulin signaling pathways which has led to the exploration and characterization of several other adipokines with similar metabolic functions (10). The fact that these cytokines inhibit the effect of insulin on endothelial cells (2) may additionally contribute to insulin resistance by limiting nutrient-induced increase in nutritive capillary flow to muscle, as well as muscle glucose uptake.

Adipokines, the bioactive mediators produced in the adipose tissue, include not only adipocytes but also other cells that are present in fat tissue. Although adipocytes can produce almost all known adipokines, pre-adipocytes, as well as macrophages and endothelial cells of adipose tissue also contribute to adipokine production. Some of these substances are specifically, or abundantly, produced by fat tissue (leptin, adiponectin, visfatin, apelin, resistin) whereas others are clearly not specific for adipose tissue since they are not exclusively or preferentially expressed there and have been previously identified and characterized as belonging to other families of substances [MCP-1, TNF- $\alpha$, IL-6, PAI-1, angiotensinogen, $11 \beta$-hydroxysteroid dehydrogenase (11 $\beta$-HSD-1, which converts inactive cortisone into active cortisol) and endocannabinoids] (11).

The adipokines, they are more than 50 different types currently recognized, act in a paracrine, autocrine and endocrine way influencing the metabolism of lipid, glucose homeostasis, in some cardiovascular risk factors, such as hypertension, as well as thrombotic and inflammatory processes. The functions of adipokines 
were recently extended far beyond metabolism to areas such as immunity, tumoral processes, angiogenesis and bone formation.

It is important to distinguish visceral from subcutaneous adipose tissue in connection with the expression of the different adipokines by their implications in the development of the metabolic syndrome. The visceral fat plays an important role in the pathogenesis of cardiovascular diseases since it expresses many components strongly involved as cardiovascular risk factors, such as IL-6, PAI-I and glucocorticoids, while the subcutaneous fat produces mostly protective substances such as leptin and adiponectin and is less sensitive to glucocorticoids (12). Furthermore, adipokines released from visceral fat have, via portal vein, a direct access to the liver, and, therefore, a huge impact on the inflammatory process.

The primary mechanisms of action of adipose tissueproduced inflammatory adipokines can be referred to according to the anatomic location of fat depot in which the adipokines are produced. Thus, adipokines released by the visceral depot would exert a greater effect on hepatic carbohydrate and lipid metabolism, stimulating hepatic release of acute phase response proteins in the liver (as CRP, an unspecific acute phase reactant that serves as an indicator of systemic inflammation) besides the autocrine/paracrine effects on the depot. Adipokines produced by the subcutaneous depot would mainly affect locally the adipose cell development and function (autocrine/paracrine effects) and exert systemic effects on, for example, skeletal muscle. The former may represent the mechanism whereby inflammatory adipokines induce hepatic insulin resistance and chronic systemic inflammation, as the latter diminish adipose tissue storage of lipids, leading to ectopic fat accumulation in the liver and skeletal muscle (13).

Many adipokines such as MCP-1, also known as C-C motif chemokine ligand 2 (CCL2), TNF- $\alpha$ and resistin (which function in humans is not clear yet), secreted by adipocytes from obese subjects can promote macrophage infiltration and accumulation in adipose tissue and subendothelial space. It has been confirmed by recent studies that showed the association of abdominal obesity with an increased amount of macrophages in adipose tissue (14). These macrophages are also implicated in the secretion of a panel of inflammatory cytokines (TNF- $\alpha$, IL-6, MCP-1, PAI- 1 ) acting in a paracrine and endocrine manner, finally causing a state of permanent low-grade inflammation in obese subjects. The MCP-1 (CCL2) receptor, C-C motif chemokine receptor 2 (CCR2), from other CC chemokine receptors present in the adipose tissue, plays a more noteworthy role in the regulation of monocyte and macrophage recruitment, besides being necessary for macrophagedependent inflammatory responses. This inflammatory state is implicated in cardiovascular disease, favoring endothelial insults and atheromatous changes (15). In Figure 1, the association between visceral adipose mass, secretion of inflammatory adipokines, insulin resistance and the metabolic syndrome is shown.

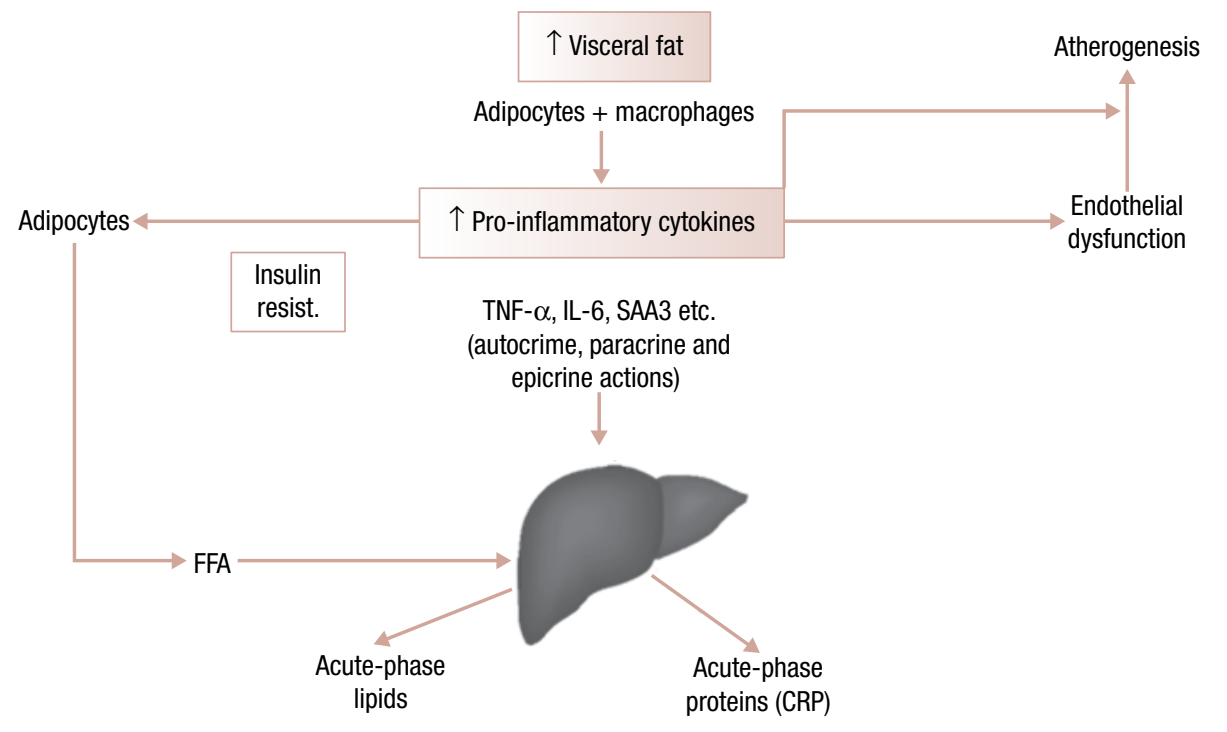

Based on Schmidt and Duncan. Clin Chem LAb Med. 2003;41:1120.

Figure 1. Association between visceral adipose mass, secretion of inflammatory cytokines, insulin resistance and the metabolic syndrome. 
Adipokines are involved in every step of the atheromatous process which begins with inflammatory changes of the vascular wall. The vascular insult is first caused by cytokines like TNF- $\alpha$ and IL- 6 , leading to increased expression of adhesion molecules such as intercellular adhesion molecule 1 (ICAM-1) and vascular cell adhesion molecule-1 (VCAM-1) which enhance monocyte adhesion to the endothelium. At the same time, MCP-1 (CCL2) secreted by adipocytes and by injured endothelial cells not only increases the migration of monocytes but also favors their transformation into foam cells. These foam cells secrete metalloproteinases that may lead to plaque rupture. Because of its low levels in obesity, the protective effects of adiponectin against plaque formation are decreased. Adiponectin is a specific white adipose tissue-derived protein, with anti-inflammatory/antiatherogenic properties such as decreasing the expression of adhesion molecules, decreasing monocyte adhesion to endothelial cells, decreasing uptake of oxidized low-density lipoprotein (LDL), decreasing foam cell formation, and decreasing proliferation and migration of vascular smooth muscle (11). Adiponectin increases insulin sensitivity, increases smooth muscle glucose uptake and free fatty acid oxidation, decreases hepatic glucose production and decreases intracellular triglycerides (16). Furthermore, obesity is also linked to a hypercoagulable state caused by increase in circulating levels of procoagulant factors such as tissue factor, fibrinogen, von Willebrand factor and factor VII. In addition, many circulating cytokines elevated in obesity will cause endothelial activation, leading to platelet activation and plug formation (17). The inhibition of fibrinolysis caused by an increased level of PAI-1 (particularly high in obesity and diabetes) is another component of this hypercoagulable process, favoring thrombus formation upon ruptured atherosclerotic plaques (18).

Hence, the state of chronic low-grade inflammation of obesity is probably the link between the different components of the metabolic syndrome and atherosclerosis.

It has been shown that distinct inflammatory pathways that occur in the adipocyte can be initiated by extracellular mediators, such as cytokines and lipids, or intracellular stresses, such as endoplasmic reticulum stress or excessive production of reactie oxygen species by the mitochondria. Signals from these mediators converge to inflammatory signaling pathways, including the kinases JNK and inhibitor of NF- $\kappa \beta$ kinase (IKK $\beta$ ). These pathways induce the production of additional inflam- matory mediators through transcriptional regulation as well as the inhibition of insulin signaling (19). Recently it has been found that different inflammatory pathways occur in the different adipose tissue depots, the omental tissue with increased NF- $\kappa \beta$ and increased JNK activity in subcutaneous abdominal adipose tissue. Additionally, NF- $\kappa \beta$ was shown to be influenced by obesity and diabetes status. These data suggest that NF- $\kappa \beta$ may play a more predominant central role in inflammatoryrelated metabolic disease in comparison to JNK (20) (Table 1). These findings are in accordance to the well known observations that visceral adipose tissue, which includes the omental tissue, is closely associated with sub-clinical inflammation, insulin resistance and type 2 diabetes mellitus (12).

\begin{tabular}{|c|c|c|}
\hline \multicolumn{3}{|c|}{ Obese non-diabetics } \\
\hline \multirow{2}{*}{ Adipose tissue depot } & JNK & NFKB \\
\hline & \multicolumn{2}{|c|}{ activities } \\
\hline Omental & $\downarrow$ & $\uparrow$ \\
\hline Abdominal subcutaneous & $\uparrow$ & \\
\hline \multicolumn{3}{|c|}{ Obese type 2 diabetics } \\
\hline Omental & & $\uparrow$ \\
\hline Abdominal subcutaneous & $\downarrow$ & \\
\hline
\end{tabular}

Source: Kusminski and cols. Diabetologia. 2007;50 Suppl 1: Abstract 153. (20)

In corroboration of genetic evidence in mice with loss of inflammatory mediators or signaling molecules prevents insulin resistance (19), pharmacological targeting of inflammatory pathways also improves insulin action. Effective treatment has been demonstrated both with inhibitors of inflammatory kinases and with agonists of relevant transcription factors. The classical drug, salicylates, in high doses, including sodium salicylate and aspirine 4 to $10 \mathrm{~g}$ of salicylate per day) was used to treat inflammatory conditions such as rheumatic fever and rheumatoid arthritis. The high doses are thought to inhibit NF- $\kappa \beta$ and its upstream activator, the IкB kinase $\beta$ (IKK $\beta$ ), as opposed to working through $c y-$ clooxygenases (COXs 1 and 2) - the classical targets of nonsteroidal anti-inflammatory drugs (NSAIDs) - in doses equivalent to $650 \mathrm{mg} /$ day of aspirine, while doses utilized in the prevention of coronary heart disease - usually from 81 to $100 \mathrm{mg} /$ day to decrease platelet aggregation - inhibit COX-1. High doses of salicylates also lower blood glucose concentrations, but are not used in present biomedical science for obvious reasons. In obese rodents and diabetic humans, high doses of 
salycilates reverse hyperglycemia, insulin resistance and dyslipidemia by sensitizing insulin signaling through inhibition of IKK $\beta$ (in liver, but not in muscle) and other possible kinases (2l).

Further research is needed to fully understand the pathophysiology of the adipokines and to determine potential targets for new therapeutic approaches. For now, it has been shown that lifestyle changes leading to weight loss are associated with an improvement of endothelial functions and reduction in circulating markers of inflammation $(22,23)$. Interestingly, weight loss by surgical removal of visceral fat, but not by subcutaneous liposuction, is associated with significant decrease in circulating CRP, IL-6, interleukin-18 (IL-18) and TNF- $\alpha$ (24). Moreover, the thiazolidinediones (TZDs), high affinity ligands of $\operatorname{PPAR} \gamma$, which are given clinically as insulin-sensitizing agents, likely improve insulin action through multiple mechanisms, including both activating lipid metabolism and reducing production of inflammatory mediators such as TNF- $\alpha$. Because of the abundant expression of PPAR $\gamma$ in adipose tissue, predominantly in subcutaneous fat, it is generally thought that the induction of adipogenesis to recruit new small adipocytes, and thus improve adipose tissue lipid accommodation and adiponectin secretion, accounts for most of the credible metabolic outcomes of thiazolidinediones (TZDs) (13), though they also have antiinflammatory actions and inhibit the transcriptional activity of TNF- $\alpha$ promoter as well as antagonize the effects of exogenous administration of TNF- $\alpha$ in vivo and in vitro, independently of the adipogenic effect of the PPAR $\gamma$ agonist (25). It was shown that short-term pioglitazone therapy $(45 \mathrm{mg} /$ day for three weeks in obese with type 2 diabetes) improved insulin sensitivity in part by reducing adipose tissue macrophage content through lowering CCL2 mRNA and CCR2 mRNA expression in subcutaneous adipose tissue, which led to a decrease of macrophage content in $69 \%$ in comparison to control, resulting in less inflammation and, then, improved insulin sensitivity (26).

In addition to these cardiovascular and metabolic effects, the chronic low grade inflammation of obesity may also play a role in other inflammatory illnesses such as joint diseases and inflammatory bowel disease. It appears to be that adipose tissue and adipokines can no longer be regarded as completely innocent in arthritis because they modulate the expression of local synovial cytokines and matrix-degrading enzymes. They may, therefore, become an attractive target for new treatment (27).
In conclusion, the adipose tissue is a highly active metabolic endocrine organ, being at the crossroads in the development of the metabolic syndrome, inflammation and atherosclerosis. Obesity and particularly visceral fat excess is associated with insulin resistance, hyperglycemia, atherogenic dyslipidemia, hypertension as well as prothrombotic and pro-inflammatory states.

Disclosure: No potential conflict of interest relevant to this article was reported.

\section{REFERENCES}

1. Reaven GM. Banting Lecture 1988. Role of insulin resistance in human disease. Diabetes. 1988;37(12):1595-607.

2. Yudkin JS. Insulin resistance and and the metabolic syndromeor the pitfalls of epidemiology. Diabetologia. 2007;50(8):1576-86.

3. Gale EA. The myth of the metabolic syndrome. Diabetologia. 2005;48(9):1679-83.

4. Pinkney J, Coppack SW, Yudkin JS. Endothelial dysfunction: cause of the insulin resistance syndrome? Diabetes. 1997;46 Suppl 2:S9-S13

5. Yudkin JS, Stehouwer CDA, Emeis JJ, Coppack SW. C-reactive protein in healthy subjects: associations with obesity, insulin resistance, and endothelial dysfunction: a potential role of cytokines originating from adipose tissue? Arterioscler Thromb Vasc Biol. 1999;19(4):972-8.

6. Wajchenberg BL, Giannella-Neto D, Silva MER, Santos RF. Depotspecific hormonal characteristics of sub-cutaneous and visceral adipose tissue and their relation to the metabolic syndrome. Horm Metab Res. 2002;34(11-12):616-21.

7. Karelis AD, St-Pierre DH, Conus F, Rabasa-Lhoret R, Poehlman ET. Metabolic and body composition factors in subgroups of obesity: what do we know? J Clin Endocrinol Metab. 2004;89(6):2569-75.

8. Stefan N, Kantartzis K, Schick F, Thamer K, Rittig K, Balletshofer $B$, et al. Identification and characterization of Metabolically benign obesity in humans. Arch Intern Med. 2008;168(15):1609-16.

9. Hotamisligil GS, Shargill NS, Spiegelman BM. Adipose expression of tumor necrosis factor-alpha: direct role in obesity-linked insulin resistance. Science. 1993;259(5091):87-91.

10. Scherer P. Adipose tissue: from lipid storage compartment to endocrine organ. Diabetes. 2006;55(6):1537-45.

11. Scherer PE. Adipose tissue: from lipid storage compartment to endocrine organ. Diabetes. 2006;55:1537-45

12. Wajchenberg BL. Subcutaneous and visceral adipose tissue: their relation to the metabolic syndrome. Endocr Rev. 2000;21(6):697-738.

13. Yang $X$, Smith $U$. Adipose tissue distribution and risk of metabolic disease: does thiazolidinedione-induced adipose tissue redistribution provide a clue to the answer? Diabetologia. 2007;50(6):1127-39.

14. Kamei N, Tobe K, Suzuki R, Ohsugi M, Watanabe T, Kubota N, et al. Overexpression of monocyte chemoattractant protein-1 in adipose tissues causes macrophage recruitment and insulin resistance. J Biol Chem. 2006;281(36):26602-14.

15. Berg $A H$, Scherer PE. Adipose tissue, inflammation, and cardiovascular disease. Circulation Res. 2005;96(9):939-49.

16. Bloomgarden ZT. Inflammation, atherosclerosis and aspects of insulin action. Diabetes Care. 2005;28(9):2312-9.

17. Davi G, Guagnano MT, Ciabattoni G, Basili S, Falco A, Marinopiccoli $\mathrm{M}$, et al. Platelet activation in obese women:role of inflammation and oxidant stress. JAMA. 2002;288(16):2008-14. 
18. Sobel BE. Increased plasminogen activator inhibitor-1 and vasculopathy. A reconcilable paradox. Circulation. 1999;99(19): 2496-8.

19. Wellen KE, Hotamisligil GS. Inflammation, stress and diabetes. J Cin Invest. 2005;115:1111-9.

20. Kusminski CM, da Silva NF, Fowler AE, Creely JS, Harte AL, Baker $A R$, et al. The effects of adiposity, diabetic status and depot-specificity on the activation of NF- $\kappa \beta$ on JNK in human abdominal adipose tissue. Diabetologia. 2007;50 Suppl 1: Abstract 0153, S69.

21. Wajchenberg BL, Nery M. Adipose tissue and inflammation. Obesity and Metabolism. 2008;4:96-8.

22. Nicoletti G, Giugliano MT, Pontillo A, Cioffi M, D’Andrea F, Giugliano D. Esposito K. Effect of a multidisciplinary program of weight reduction on endothelial function in obese women. J Endocrinol Invest. 2003;26(3):RC5-8.

23. Heilbroon LK, Noakes M, Clifton PM. Energy restriction and weight loss on very-low-fat diets reduce $\mathrm{C}$-reactive protein concen- trations in obese, healthy women. Arterioscl Thromb Vasc Biol. 2001;21(6):968-70.

24. Klein S, Fontana L, Young VL, Coggan AR, Kilo C, Patterson BW, Mohammed BS. Absence of effect of liposuction on insulin action and risk factors for coronary heart disease. N Engl J Med. 2004;350(25):2549-57.

25. Lee $\mathrm{CH}$, Olson P, Evans RM. Mini-review: lipid metabolism, metabolic diseases, and peroxisome proliferators-activated receptors. Endocrinology. 2003;144(6):2201-7.

26. Lee D-E, Kishore P, Li W, Zhang K, Schiwek, Hawkins M, Saper. The effect of short-term pioglitazone therapy on CCR-1 expression in subcutaneous adipose tissue in subjects with T2DM. Program \& Abstracts. The Endocrine Society $89^{\text {th }}$ Annual Meeting, 2007 June 2-5; OR 28, p. 111.

27. Schaffler A, Muller-Ladner U, Scholmerich J, Bucher C. Role of adipose tissue as an inflammatory organ in human disease. Endocr Rev. 2006;27(5):449-67. 